\title{
Model prediction of impurity retention in stochastic magnetic boundary and comparison with edge carbon emission in LHD
}

\author{
M. Kobayashi ${ }^{a^{*}}$, Y. Feng ${ }^{b}$, S. Morita ${ }^{a}$, M.B. Chowdhuri ${ }^{a}$, M. Goto ${ }^{a}$, S. Masuzaki ${ }^{a}$, N.

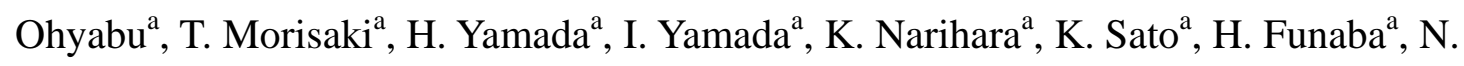 \\ Tamura $^{\mathrm{a}}$, Y. Nakamura ${ }^{\mathrm{a}}$, A. Komori ${ }^{\mathrm{a}}$, O. Motojima ${ }^{\mathrm{a}}$ and the LHD experimental group \\ ${ }^{a}$ National Institute for Fusion Science, 322-6 Oroshi-cho, Toki 509-5292, Japan \\ ${ }^{b}$ Max-Planck-Institute fuer Plasmaphysik, Wendelsteinstrasse 1, D-17491 Greifswald, Germany
}

\begin{abstract}
Impurity retention (screening) potential in the edge region with stochastic magnetic field in LHD (Large Helical Device) is studied numerically using the three dimensional edge transport code, EMC3-EIRENE, and experimentally applying VUV and EUV spectrometers. The code predicts an impurity retention regime at high densities, where the friction force dominates over the thermal force. The carbon radiation intensities from each charge state are measured in experiments, and compared with the modelling results. The density dependencies of the calculated and measured radiation intensities agree well with each other, confirming the impurity retention in the edge region of LHD. Geometrical advantages of the stochastic field line structure in this concern are also discussed.
\end{abstract}

PACS:52.55.Fa, 52.55.Rk, 52.55.Fi, 52.65.Pp

JNM keywords: I0100 Impurities, P0500 Plasma-Materials Interaction

PSI-18 keywords: Impurity transport, Divertor modelling, 3D edge plasma

*Corresponding author address: 322-6 Oroshi-cho, Toki 509-5292, Japan

*Corresponding author E-mail : kobayashi.masahiro@lhd.nifs.ac.jp

Presenting author: Masahiro Kobayashi

Presenting author e-mail : kobayashi.masahiro@lhd.nifs.ac.jp 


\section{Introduction}

This paper presents an impurity transport analysis in the edge region with stochastic magnetic field in LHD by applying three dimensional modelling and a comparison with carbon emission measurements, in order to identify impurity retention (screening) effect. M.Z. Tokar has analytically shown the importance of background-plasma flow for impurity exhaust from stochastic boundary plasma [1]. From the parallel momentum equation for impurity [2], dominating terms are found to be friction force and ion thermal force. The force balance between the two terms determines impurity velocity along field lines,

$$
V_{z / /} \approx V_{i / /}+C_{i} \frac{\tau_{s}}{m_{z}} Z^{2} \nabla_{/ /} T_{i}
$$

where $V_{\mathrm{z} / /}, \mathrm{V}_{\mathrm{i} / /}, \mathrm{T}_{\mathrm{i}}$ are parallel flow of the impurity, of background plasma and ion temperature. $\tau_{\mathrm{s}}, \mathrm{m}_{\mathrm{z}}$ and $\mathrm{Z}$ represent impurity-ion collision time, impurity mass and charge states, respectively. $C_{i}$ is a numerical factor. The first and the second terms on the right hand side are contributions from the friction and the ion thermal force, respectively. The condition to have friction dominant case is [3] [4],

$$
\frac{\text { friction force }}{\text { thermal force }} \sim \frac{5 / 2 n_{i} T_{i} V_{i / /}}{\kappa_{i}^{0} T_{i}^{2.5} \nabla_{/ /} T_{i}}>1 \text {. }
$$

The possibility of impurity exhaust by friction force with increasing density was discussed in the LHD local island divertor configuration using parallel 1D modelling [5]. 3D edge transport simulation with EMC3 [6] coupled with EIRENE [7] in W7-AS has predicted impurity retention in the divertor region by friction force at high density discharge [8]. The recent experimental and numerical analysis in Tore Supra showed reduction of core carbon content with decreasing edge temperature [9]. The purpose of this paper is to obtain direct comparison between modelling analysis and experimental observations in order to identify the existence of impurity retention effect by the friction force. 3D impurity transport modelling analysis using EMC3-EIRENE has been carried out in LHD [10]. In the next section, the modelling results are briefly revisited to outline the mechanism of the impurity retention in 
the edge region with stochastic magnetic field. In section 3, the modelling results are compared with experiments and a summary is given in section 4 .

\section{3D numerical impurity transport analysis}

In the 3D modelling, the stochastic field line trajectories in the edge region are accurately reproduced in the code using the reversible field-line mapping (RFLM) technique [11]. The realistic three dimensional geometry of the vessel wall/divertor plates is also precisely treated in the code. The conservation form of Braginskii equation [12] of mass, momentum and energy is solved by Monte Carlo scheme. The computational domain starts from last closed flux surface (LCFS) and covers the entire edge region. The background plasma is calculated by adjusting the anomalous cross-field transport coefficients so as to reproduce edge temperature and density profiles obtained in experiments [13]. Upstream density, $\mathrm{n}_{\text {LCFS }}$, and the energy flux through the LCFS are specified as boundary conditions. The both parameters are obtained from experiments. Bohm boundary condition is applied at the divertor plates. In the present analysis, carbon is selected as impurity, which is main impurity species in LHD because the divertor plates are made of carbon. The neutral carbon is released from the divertor plates according to plasma particle flux distribution. Sputtering coefficient is fixed to 0.02 for the presented computations. The details of physics model and application at LHD configuration of the code are found in ref. [13] [14]. $\mathrm{n}_{\text {LCFS }}$ is scanned while input power is fixed to $8 \mathrm{MW}$.

The obtained carbon density profiles are integrated over poloidal and toroidal directions and plotted as a function of radial coordinate, $r_{\text {eff }}$, which is defined by a constant volume in cylindrical approximation enclosed by each radial surface of the computational mesh, figure 1 (a). Connection length distribution in the edge region obtained by the RFLM technique mentioned above is also shown in figure 1 (b). The figure is superposed with Poincare plot to identify remnant islands. The outer-most region is characterized with mixture of long and 
short connection lengths flux tubes, which is created by stretch and bend of flux tubes due to magnetic shear. The region is called edge surface layers [15]. The similar structure is also seen in stochastic boundary of other machines $[16,17]$. The inner part, $\mathrm{r}_{\mathrm{eff}}<0.65 \mathrm{~m}$, is called stochastic region, where connection length becomes more than several hundred meters and remnant island structure is observed with white color in the figure.

The outer most part, $\mathrm{r}_{\mathrm{eff}}>0.70 \mathrm{~m}$, is found to be always in friction force dominant phase due to low temperature and acceleration of plasma flow near to the divertor. This is reflected by positive gradient of carbon density profiles for all densities. At the low density case, $\mathrm{n}_{\mathrm{LCFS}}$ $=2.0 \times 10^{19} \mathrm{~m}^{-3}$, as moving to inner radius, where temperature is raised up to $100 \sim 200 \mathrm{eV}$, the carbon density starts to increase due to strong thermal force. The impurity builds up at LCFS with $n_{\text {ILCFS }}>n_{I}(a)$. With increasing density, the friction dominant region gradually extends to the inner radius. At $\mathrm{n}_{\mathrm{LCFS}}=4 \times 10^{19} \mathrm{~m}^{-3}$, the inward flux of impurity disappears in the entire edge region with $n_{\text {ILCFS }}<n_{I}(a)$. In the edge surface layer, the short flux tubes, which act as particle and heat sink, accelerate plasma flow as well as reduces temperature, effectively. This easily creates situation favourable for impurity retention when density is raised. The positive gradient of carbon at $\mathrm{r}_{\text {eff }}>0.68 \mathrm{~m}$ is clear indication of outward impurity flow. In the stochastic region, $\mathrm{r}_{\mathrm{eff}}<0.65 \mathrm{~m}$, on the other hand, the impurity build up disappears, resulting in a flat profile. This is found to be a geometrical advantage of the stochastic region in LHD. As discussed in ref. [13][14], plasma transport follows the remnant island structures in the stochastic region, radial energy flux, $\mathrm{q}_{\mathrm{r}}$, can be written as,

$$
q_{r}=-n \chi_{\perp} \frac{\partial T}{\partial r}-\kappa_{/ /} T^{2.5} \frac{\partial T}{\partial l} \vec{b} \cdot \vec{r},
$$

where $\chi_{\perp}$ is cross field heat diffusivity and $\kappa_{/ /}=6.5 \times 10^{20}\left(\mathrm{~m}^{-1} \mathrm{~s}^{-1} \mathrm{eV}^{-2.5}\right)$ for ion. $l, \vec{b}, \vec{r}$ are a coordinate along field lines, a local base vector tangential to field line and one in radial direction, respectively. The first term is a cross-field heat conduction and the second term is a projection of parallel heat flux onto radial direction caused by braiding magnetic field. This 
can be written as

$$
q=-n \chi_{\perp} \frac{d T}{d r}-\Theta^{2} \kappa_{/ /} T^{5 / 2} \frac{d T}{d r}
$$

, where the parallel derivative is replaced with radial one with $\frac{\partial}{\partial l}=\vec{b} \cdot \vec{r} \frac{\partial}{\partial r}$, and $\Theta=\vec{b} \cdot \vec{r}$. $\Theta$ corresponds to the field line pitch inside the remnant islands in the stochastic region, defined as $d r / d l$ with $d r$ being a radial extension of the islands and $d l$ a parallel length to circumference inside the island, respectively. The condition for the cross field energy flux (first term) to dominate over parallel one is given as [8],

$$
\frac{n}{T_{i}^{5 / 2}}>\frac{\Theta^{2} \kappa_{\|}^{i}}{\chi_{\perp}^{i}}
$$

The smaller $\Theta$ is, the larger the cross field transport contributions becomes. In LHD, $\Theta$ is estimated as $\sim 10^{-4}$. For typical edge plasma parameters in LHD, $T_{i} \sim 100 \mathrm{eV}, n \sim 10^{19} \mathrm{~m}^{-3}$ with $\chi_{\perp} \sim 1 \mathrm{~m}^{2} / \mathrm{s}$, the condition is easily satisfied, $10^{-5}>6.5 \times 10^{-7}$. Since the cross field energy flux replaces the parallel one by short-cutting the islands, the parallel temperature gradient is suppressed. Therefore, as shown in eq.(2), increasing density at the same time decreasing temperature with fixed input power leads to friction dominant regime.

\section{Indication of impurity retention in experiments}

Plotted in figure 2 are radial density profiles of each charge state in case of $n_{\text {LCFS }}=2$ and $4 \mathrm{x}$ $10^{19} \mathrm{~m}^{-3}$. When the retention occurs, carbon is flushed out of the edge region and builds up at around the edge surface layers. Thereby the population of lower charge states increases as shown in the figure. In addition to this, due to the large difference of ionization potential of carbon between $\mathrm{C}^{3+}(=64.5 \mathrm{eV})$ and $\mathrm{C}^{4+}(=392 \mathrm{eV})$, there is clear separation in the radial profile of lower and higher charge states. $\mathrm{C}^{+}$to $\mathrm{C}^{3+}$ localize at $\mathrm{r}_{\text {eff }}>0.68 \mathrm{~m}$, while $\mathrm{C}^{4+}$ to $\mathrm{C}^{6+}$ exist mainly at $\mathrm{r}_{\text {eff }}<0.68 \mathrm{~m}$. In case of low plasma density, on the contrary, the population of higher charge states increases because of the build up around LCFS. Such a change of population will be reflected on the radiation intensity from each charge states, and this can be 
directly compared with experimental observations.

In the experiments, carbon line radiation is measured with VUV monochromators and EUV spectrometer [18] [19]. The following lines are selected for each charge state: CIII

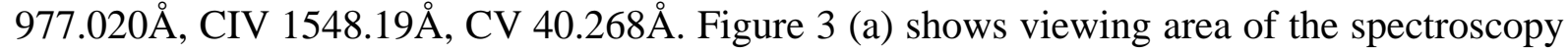
relative to the plasma, where the shape of the flux surfaces and the divertor legs are indicated. Figure 3 (b) shows CIII radiation distribution in the plane obtained by the code. The viewing area covers the edge surface layer as well as the stochastic region, and we have confirmed that the coverage of the view angle is large enough to detect the emissivity change caused by the retention of carbon observed in the code. Figure 4 (a) plots the measured intensity of CIII, CIV and CV normalized with density as a function of line averaged density. There is clear difference in the behavior between lower and higher charge states. With increasing density the CIII and CIV intensities increase, while that of CV decreases. Since the emissivity of CIV is insensitive to temperature in the range of 10 to $100 \mathrm{eV}$, this indicates increase of $\mathrm{C}^{2+}$ and $\mathrm{C}^{3+}$ densities. In contrast, CV emissivity does have substantial temperature dependence in a range of several hundred eV. Nevertheless, no remarkable change in edge Te-profiles during the density scan is observed by Thomson scattering system which has a high accuracy in Te-measurement. Thus, the observed reduction in CV emission reflects actually a decrease in $\mathrm{C}^{4+}$ density.

Emission intensities obtained from the modelling are plotted in figure 4 (b), where trend against the density is in good agreement with experiments, i.e. the increase of CIII and CIV and the simultaneous decrease of CV. As shown in the previous section and in the last paragraph, such a density dependence of radiation intensity is caused by a change in the force balance. Retention occurs when friction becomes dominating. For comparison, simulation results in the absence of background plasma flows in the parallel impurity momentum equation are also plotted in the figure, where the carbon always accumulates around LCFS due to dominant thermal force. Because of no outward convection of impurity, the carbon 
density continuously increases with increasing source which is proportional to divertor flux in the present analysis. The deviation of CIII and CIV from experiments becomes large by more than one order of magnitude. CV radiation starts to increases monotonically, showing opposite trend against experiments. From this comparison, it is found that the friction force has significant effect on the density dependence of radiation intensity. The results indicate impurity retention in the edge region with stochastic magnetic field caused by the increasing friction force and suppression of thermal force at higher density.

\section{Summary}

3D numerical impurity transport study has been performed in the edge region with stochastic magnetic field in LHD. The impurity transport modelling predicts impurity retention at higher density regime. The mechanism of the retention is summarized as follows : With increasing background plasma density, the friction term in the parallel impurity momentum transport equation becomes dominant over the thermal force especially in the edge surface layer, where the low temperature and the acceleration of background plasma flow towards divertor are most effective to drives the impurity to divertor region by the enhanced friction force. At the same time, the increased cross-field energy flux across the remnant islands in the stochastic region replaces the parallel one. This then suppress the parallel temperature gradient, thereby thermal force, resulting in the flat impurity density profile there (figure 1 (a)). This regime is achieved by the geometrical feature of the stochastic region of LHD with very small field line pitch inside the island, $\Theta \sim 10^{-4}$.

The similar experimental observation of carbon radiation intensity of CIII and CV to those presented here is reported during activation of stochastic field in TEXTOR-DED experiments [20]. The transport analysis of the laminar zone conducted in ref. [16, 17] indicates also similarity to ones of the edge surface layers in LHD. The mechanism suggested in the present work could, therefore, be universal feature in a stochastic magnetic boundary. 
Further inter-machine analysis will be necessary to clarify this point.

\section{Acknowledgments}

The authors are grateful for the support for computational resource, AMD Opteron-InfiniBand

Cluster Machine, by Grant-in-Aid for Science Research on Priority Areas (Area 465, No.18070005) from Ministry of Education, Culture, Sports, Science and Technology, Japan. The part of the computational work is also financially supported by the budget code NIFS08ULPP538.

\section{References}

[1] M.Z. Tokar, Phys. Plasmas 6 (1999) 2808.

[2] P.C. Stangeby and J.D. Elder, Nucl. Fusion 35 (1995) 1391.

[3] Yu.L. Igitkhanov, Contrib. Plasma Phys. 28 (1988) 477.

[4] S.I. Krashenninikov et al., Nucl. Fusion 31 (1991) 1455.

[5] M. Kobayashi et al., Contrib. Plasma Phys. 46 (2006) 527.

[6] Y. Feng et al., Contrib. Plasma Phys. 44 (2004) 57.

[7] D. Reiter et al., Fusion Sci. Technol. 47 (2005) 172.

[8] Y. Feng et al., Nucl. Fusion 46 (2006) 807.

[9] Y. Corre et al., Nucl. Fusion 47 (2007) 119.

[10] M. Kobayashi et al., Contrib. Plasma Phys. 48 (2008) 255.

[11] Y. Feng et al., Phys Plasmas 12 (2005) 052505.

[12] S.I. Braginskii, in Review of Plasma Physics, edited by M. Leontovich (Consultants Bureau, New York, 1963), Vol. 1, p.205.

[13] M. Kobayashi et al., J. Nucl. Mater. 363-365 (2007) 294.

[14] Y. Feng et al., Nucl. Fusion 48 (2008) 024012.

[15] N. Ohyabu et al., Nucl. Fusion 34 (1994) 387. 
[16] F. Nguyen et al., Nucl. Fusion 37 (1997) 743.

[17] O. Schmitz et al., Nucl. Fusion 48 (2008) 024009.

[18] M.B. Chowdhuri, S. Morita and M. Goto et al., Rev. Sci. Instrum. 78 (2007) 023501.

[19] M.B. Chowdhuri, S. Morita and M. Goto, Appl. Optics 47 (2008) 135.

[20] G. Telesca et al, “Carbon transport in the stochastic magnetic boundary of TEXTOR”, in this conference. 


\section{Figure captions}

Fig. 1 : (a) Carbon density profiles for different densities, obtained by EMC3-EIRENE. The density is integrated over poloidal and toroidal directions. $r_{\text {eff }}$ is defined by cylindrical approximation of the volume enclosed by each radial surface of computational mesh. The density is normalized with those at outer most boundary of the edge region, $\mathrm{n}_{\mathrm{I}}(\mathrm{a})$ (b) Connection length profile in the edge region of LHD, plotted in $\mathrm{r}_{\mathrm{eff}}$ and poloidal coordinates.

Fig.2 : Radial carbon density profiles of each charge state, obtained by EMC3-EIRENE with $\mathrm{n}_{\mathrm{LCFS}}=$ (a) $2 \times 10^{19}$ and (b) $4 \times 10^{19} \mathrm{~m}^{-3}$, input power of 8MW.

Fig.3 : (a) The viewing area of spectroscopy measurements relative to the plasma, where the shape of flux surfaces and the divertor legs of the plasma are indicated. The spectroscopy collects the emission from the region between the two horizontal dashed lines. (b) The CIII radiation distribution in the same plane as (a), obtained by the 3D modelling.

Fig. 4 : (a) Emission of CIII, CIV and CV obtained in experiments normalized with line averaged density as a function of the density. (b) Emission of CIII, CIV and CV calculated from the 3D modeling. $\mathbf{\square}$ : CIII, $\bigcirc$ : CIV and $\diamond$ CV. Solid, dashed and doted lines in (b) are CIII, CIV and CV without friction force obtained in the modeling. 
Fig. 1 M. Kobayashi et al.
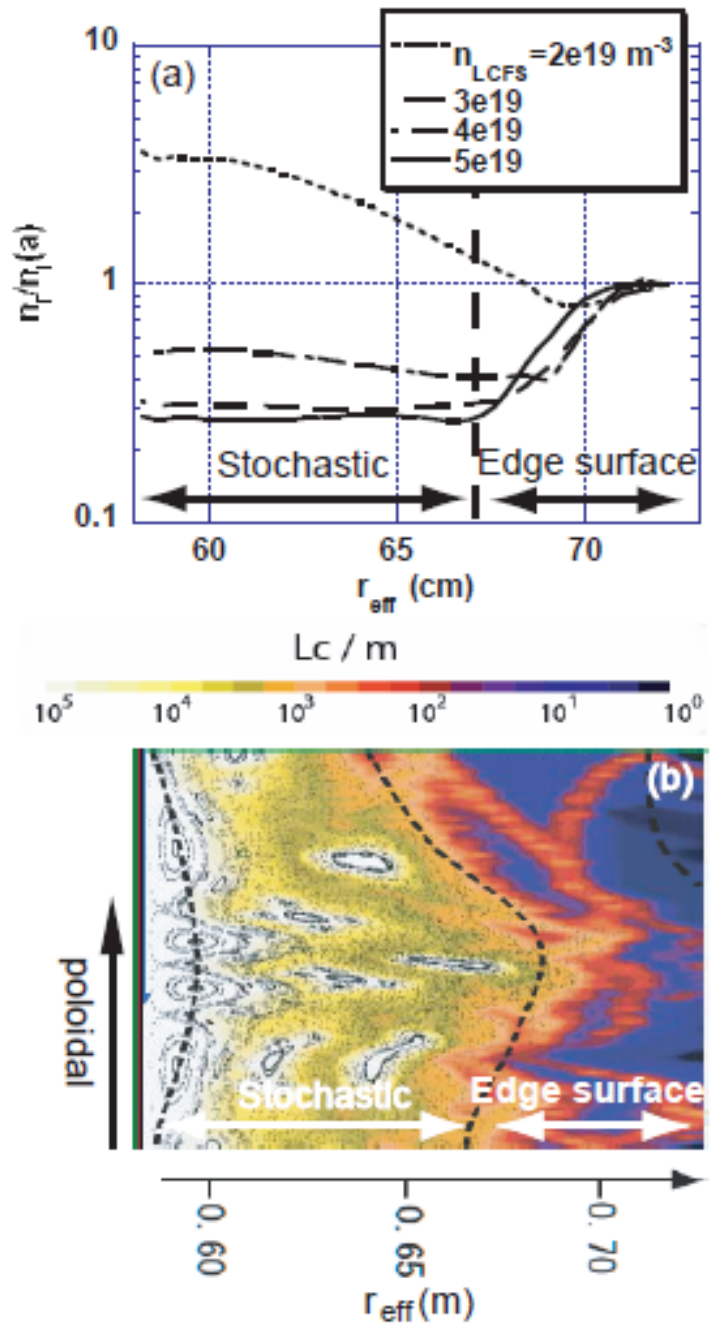
Fig. 2 M. Kobayashi et al.
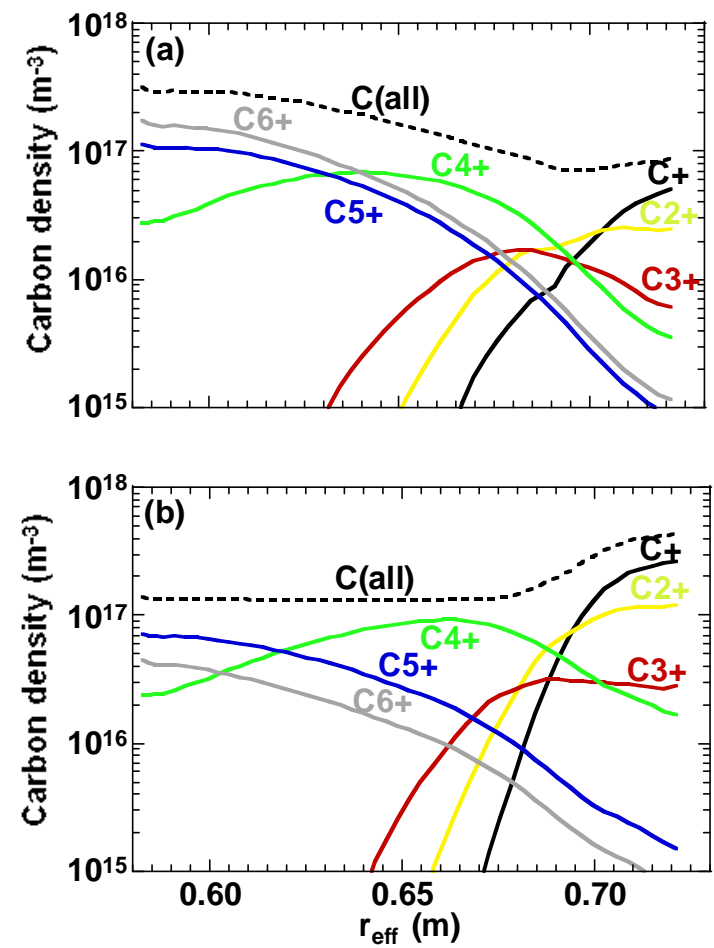
Fig.3 M. Kobayashi et al.
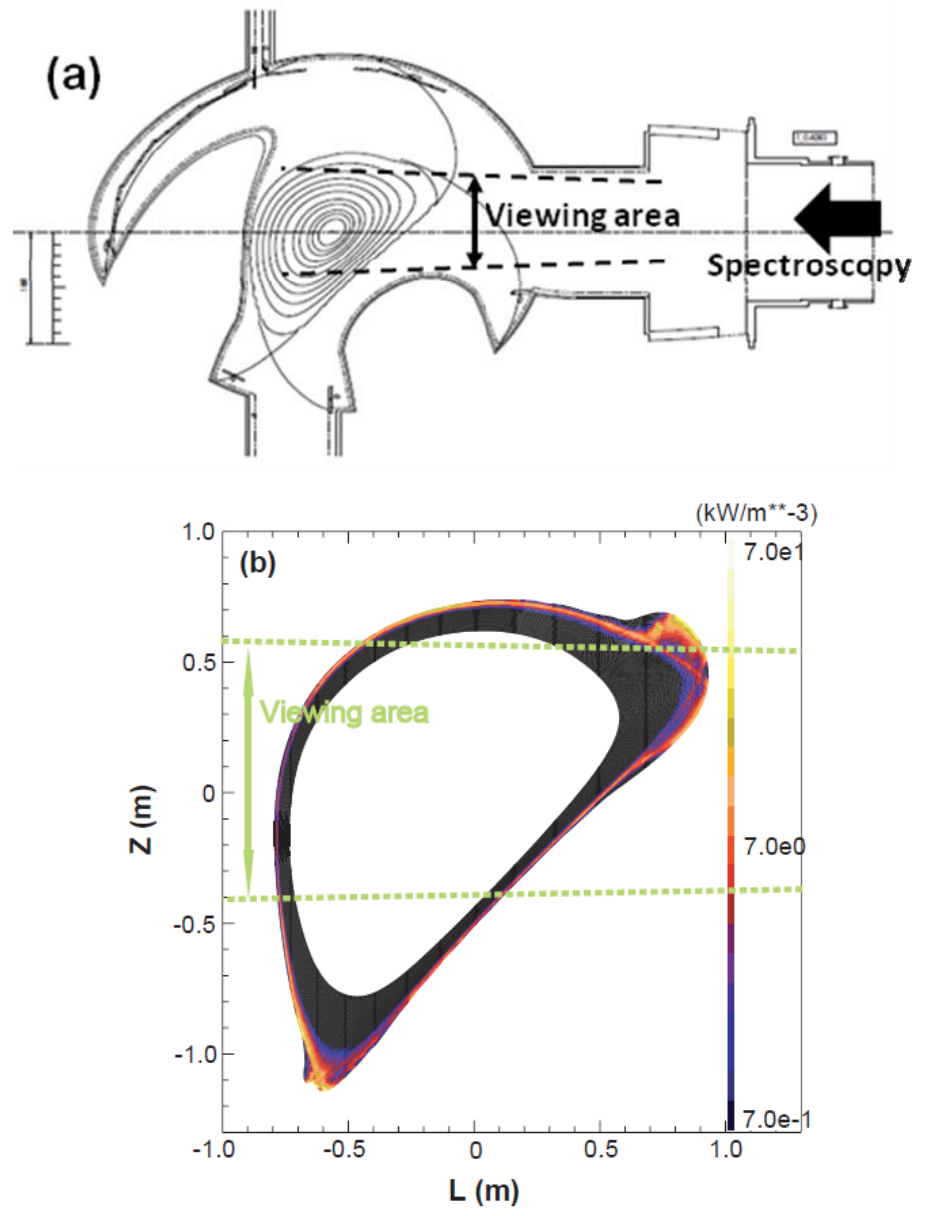
Fig.4 M. Kobayashi et al.
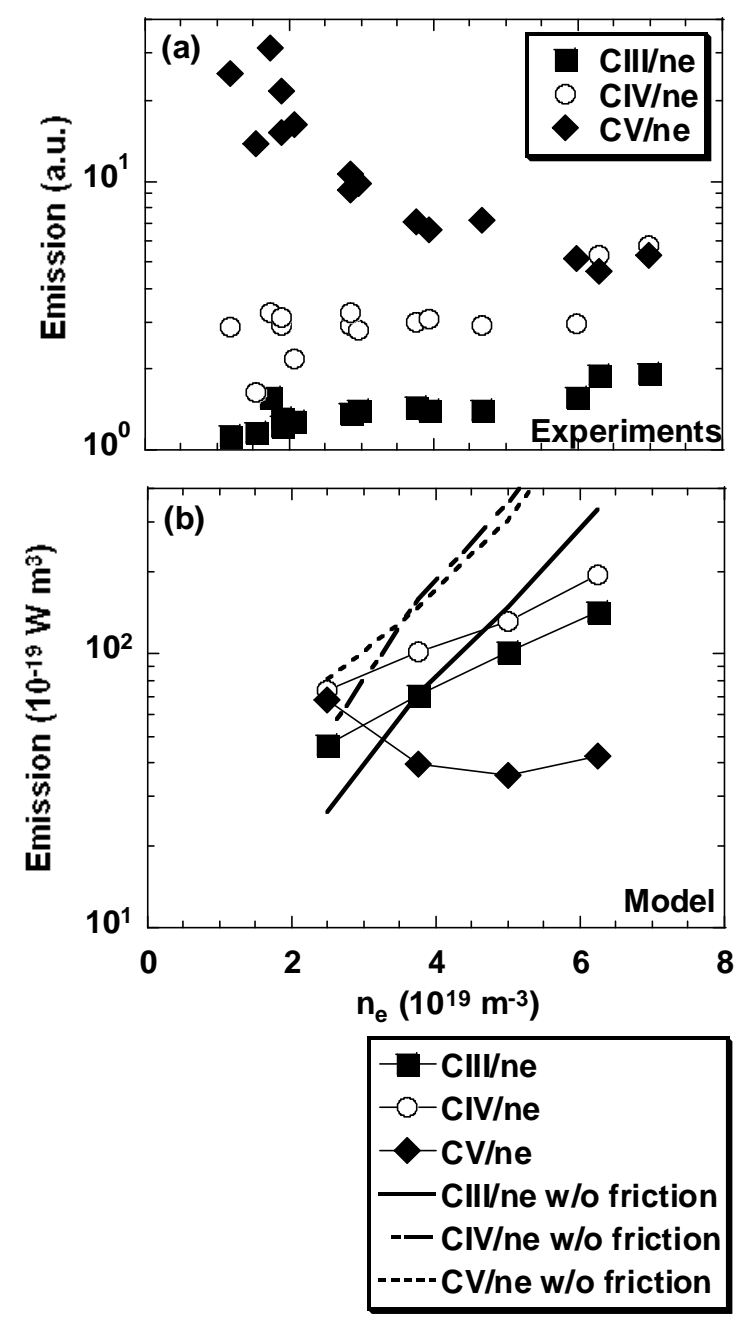